Les lasers à impulsions

ultra-brèves permettent

aujourd'hui d'atteindre des

éclairements lumineux

extrêmement élevés, tels que

les électrons d'une cible placée

au foyer du faisceau acquièrent

en quelques femtosecondes

des vitesses relativistes.

Ce régime $d^{\prime}$ interaction extrême

pose de nombreuses questions

d'ordre fondamental. II s'agit

notamment de déterminer

ce que deviennent les lois

de l'optique à ces intensités.

Nous utilisons ici l'exemple

des miroirs plasmas pour

illustrer cette problématique

et les phénomènes physiques

mis en jeu, et montrer comment

ce nouveau régime de l'optique permet d'obtenir de nouvelles

sources de lumière aux

propriétés remarquables,

très prometteuses en termes

d'applications.

\title{
Vers l'optique à ultra-haute intensité : l'exemple des miroirs plasmas
}

Fabien Quéré (fabien.quéré@cea.fr) et Philippe Martin

DSM/IRAMIS, Service des Photons, Atomes et Molécules, CEA/Saclay, 91191 Gif-sur-Yvette Cedex

\section{Lasers ultra-brefs et intensités lumineuses extrêmes}

La technologie laser permet aujourd'hui de produire des impulsions de lumière extrêmement brèves, pouvant atteindre des durées de quelques femtosecondes $\left(1 \mathrm{fs}=10^{-15} \mathrm{~s}\right)$, correspondant à seulement quelques cycles optiques dans la gamme visible ou proche visible. Un des intérêts majeurs de ces impulsions ultra-brèves de lumière cohérente est de permettre de concentrer très fortement l'énergie électromagnétique, temporellement et spatialement : on atteint ainsi des éclairements $\mathrm{I}=\mathrm{E} /\left(\pi \mathrm{r}^{2} \tau\right)$ (E et $\tau$ énergie et durée de l'impulsion, $r$ diamètre de la tache focale) extrêmement élevés avec des quantités d'énergie par impulsion relativement modérées, et donc des lasers de dimensions modestes. Les lasers ultra-brefs les plus puissants délivrent aujourd'hui typiquement des impulsions d'une énergie de quelques joules, avec des durées de quelques dizaines de fs (cf. encadrés 1 et 2 ) : on obtient donc des puissances crêtes $\mathrm{P}=\mathrm{E} / \tau$ de l'ordre de quelques centaines de terawatt $\left(1 \mathrm{TW}=10^{12} \mathrm{~W}\right)$ et, après focalisation du faisceau sur quelques $\mu \mathrm{m}^{2}$, des éclairements lumineux I de plus de $10^{20} \mathrm{~W} / \mathrm{cm}^{2}[1,2]$.

Lorsqu'une cible est soumise à un champ laser d'une telle intensité, la vitesse des électrons de cette cible, acquise sous l'effet du champ électromagnétique, devient de l'ordre de la vitesse de la lumière, c : on entre ainsi dans un nouveau régime d'interaction laser-matière, qualifié de relativiste (cf. encadré 3, p. 16). Les lois de l'optique linéaire et non linéaire sont bien connues jusqu'à des intensités de quelques $\mathrm{TW} / \mathrm{cm}^{2}$; une question d'ordre fondamental est de savoir ce que deviennent ces lois aux intensités lumineuses encore plus fortes, et notamment dans ce régime relativiste.

\section{Les lasers ultra-intenses ultra-brefs}

Les lasers ultra-intenses sont actuellement tous basés sur le même principe technique, I'amplification à dérive de fréquence, démontré expérimentalement par D. Strickland et G. Mourou en 1985.

Le début de la chaîne laser consiste en un oscillateur à modes bloqués (cf. encadré 2), délivrant des impulsions femtosecondes, de quelques nanojoules d'énergie seulement. Le problème qui se pose est alors d'amplifier ces impulsions sans dégrader la qualité spatiale et temporelle du faisceau, et surtout sans endommager de façon irréversible des éléments optiques de la chaîne. Pour éviter ces problèmes, on réduit l'intensité des impulsions injectées dans la chaîne, en les étirant temporellement, d'un facteur typiquement $10^{4}$ (jusqu'à quelques centaines de ps), tout en conservant le même spectre. Ceci est réalisé en utilisant un système optique dispersif pour étaler temporellement les différentes fréquences comprises dans ce spectre, c'est-à-dire pour induire une dérive temporelle de fréquence. Ces impulsions étirées, d'intensité réduite, sont ensuite amplifiées jusqu'à plusieurs joules, par plusieurs dizaines de passages dans des milieux lasers présentant du gain sur une large gamme spectrale. Après amplification, le faisceau est finalement recomprimé à des durées de quelques dizaines de femtosecondes, en synchronisant à nouveau le mieux possible toutes les fréquences comprises dans le spectre. 


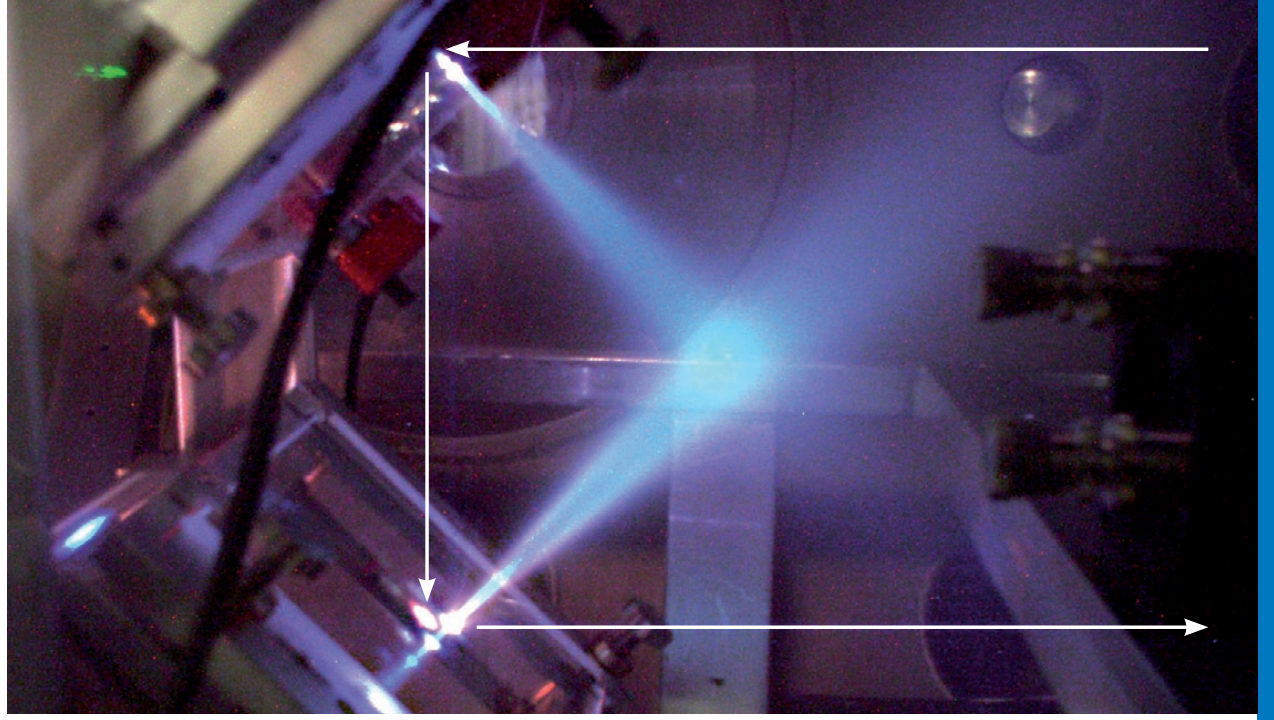

1. Photo d'un double miroir plasma en fonctionnement. Le trajet du faisceau laser est indiqué par les flèches blanches. Les deux lames diélectriques utilisées comme miroir plasma sont sur la gauche de l'image. Les deux faisceaux lumineux bleutés correspondent à l'émission de lumière par les plasmas en cours d'expansion, créés initialement à la surface de ces lames par l'impulsion laser. Courtoisie: : Jan-Paul Geindre, LUL.

Cette question complexe est loin d'être totalement élucidée : quelques-uns des principaux phénomènes physiques mis en jeu ont néanmoins été identifiés ces dernières années, comme nous allons le voir dans cet article. C'est notamment le cas pour l'interaction avec un " miroir plasma ", qui est un plasma dense créé par ionisation d'une cible solide par une impulsion ultrabrève intense.

\section{Notion de miroir plasma}

Les miroirs sont les composants les plus élémentaires et les plus utilisés en optique. Grâce aux impulsions lasers ultra-brèves, il est possible d'étudier leur comportement physique à très haute intensité, mais également de les utiliser comme éléments optiques actifs pour obtenir des sources de lumière aux propriétés remarquables.
Lorsqu'une impulsion laser ultra-brève est focalisée à une intensité suffisamment élevée (typiquement $>10^{15} \mathrm{~W} / \mathrm{cm}^{2}$ ) sur une cible solide, cette dernière se trouve fortement ionisée par le champ électromagnétique intense en un temps très court, durant le front montant de l'impulsion. Un plasma est ainsi créé en surface de la cible. Du fait de la brièveté de l'impulsion, ce plasma n'a pas le temps de se détendre de façon significative vers le vide pendant l'impulsion, et l'interface plasma-vide peut donc rester extrêmement abrupte (largeur $\mathrm{L}<<\lambda$, où $\lambda$ est la longueur d'onde du laser) durant toute l'interaction. On obtient donc un plasma dense (c'est-à-dire de densité $\mathrm{n}>>\mathrm{n}_{\mathrm{c}}=\left(\mathrm{m \varepsilon _{0 }} / \mathrm{e}^{2}\right) \omega^{2}$, où $\mathrm{n}_{\mathrm{c}}$ est la densité critique au-delà de laquelle l'onde laser de fréquence $\omega$ ne peut se propager dans le plasma), qui se comporte comme un miroir de qualité optique pour l'impulsion laser ultra-brève : celle-ci est alors réfléchie de façon spéculaire par ce "miroir plasma ", et la qualité spatiale du faisceau est préservée après réflexion. Étant fortement ionisé par nature, un miroir plasma peut être exposé à des champs lasers ultra-intenses, jusqu'au régime d'interaction relativiste.

\section{Le problème du contraste temporel}

Les lasers permettant d'atteindre le régime d'interaction relativiste existent depuis bientôt 15 ans. Néanmoins, ce n'est que durant ces 5 dernières années que la physique des miroirs plasmas à très haute intensité a pu faire l'objet d'études expérimentales suffisamment approfondies pour en améliorer la compréhension. Ce décalage est lié à un problème technique majeur ayant longtemps fait obstacle : celui du contraste temporel des lasers ultra-intenses.

\section{Comment produire des impulsions ultra-brèves?}

Considérons une source de lumière dont le spectre $\mathrm{s}(\omega)=|\mathrm{E}(\omega)|$ (supposé ici de forme gaussienne) a une largeur $\Delta \omega$ (définie comme l'écart-type du spectre). D'après les relations d'incertitude temps-fréquence découlant des propriétés de la transformée de Fourier, l'impulsion la plus courte qui puisse être obtenue à partir d'un tel spectre a une durée $\Delta \tau=1 /(2 \Delta \omega)$ (où $\Delta \tau$ est l'écart-type de la fonction $E(t)$ ). On obtient cette impulsion lorsque toutes les fréquences du spectre $E(\omega)$ sont parfaitement en phase, c'est-à-dire lorsque la phase spectrale $\varphi(\omega)=\operatorname{Arg} E(\omega)$ est constante - on parle alors de fréquences bloquées en phase. Une source d'impulsions ultra-brèves doit donc remplir ces deux conditions : (1) générer un spectre électromagnétique large, et cela avec (2) une « bonne » relation de phase entre les différentes fréquences - l'optimum étant une phase spectrale constante, permettant d'obtenir une impulsion " limitée par transformée de Fourier », de durée $\Delta \tau=1 /(2 \Delta \omega)$.

Dans les sources lasers ultra-brèves, la condition (1) implique d'utiliser un milieu laser présentant une courbe de gain spectralement large, adaptée aux durées que l'on souhaite obtenir. La condition (2) est réalisée en utilisant des effets non linéaires pour assurer le fonctionnement du laser en mode d'impulsions courtes (donc d'intensité élevée), et en contrôlant avec soin la dispersion de tous les éléments optiques utilisés. On parvient ainsi aujourd'hui à produire des impulsions de quelques femtosecondes en sortie d'un oscillateur, soit quelques cycles optiques seulement, dans le visible ou le proche visible.

Le passage à des durées d'impulsion attosecondes (as) devient beaucoup plus problématique : une impulsion de 100 as a une largeur spectrale supérieure à $20 \mathrm{eV}$, qu'il est pour l'heure absolument impossible d'obtenir avec des milieux amplificateurs. Pour obtenir de telles largeurs spectrales, on utilise donc l'optique non linéaire, et en particulier la génération d'harmoniques d'ordre très élevé de faisceaux lasers intenses. L'un des principaux problèmes est alors de conserver une bonne relation de phase entre ces différentes fréquences.. 


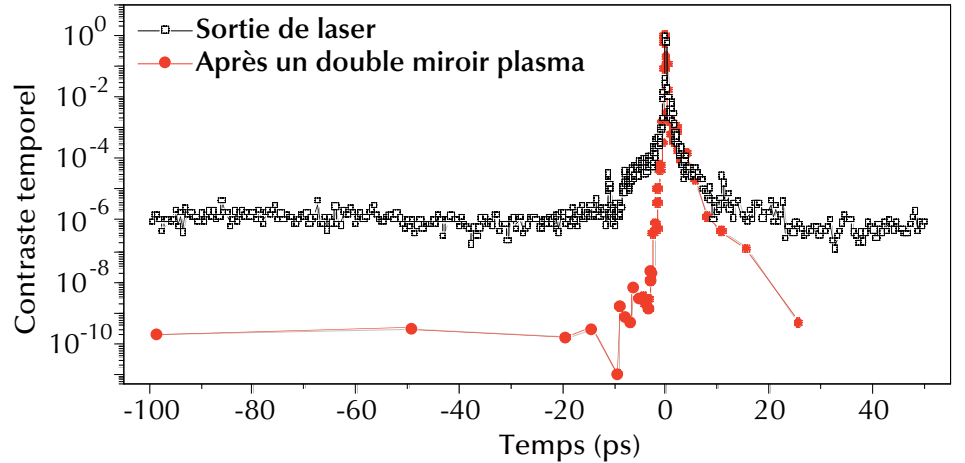

2. Profil temporel d'un laser $10 \mathrm{TW}-60$ fs en échelle logarithmique, avec et sans double miroir plasma. Les deux miroirs plasmas utilisés en série permettent de réduire de 4 ordres de grandeur environ l'intensité du piédestal précédant l'impulsion principale. La partie du piédestal suivant l'impulsion principale est également réduite, dans une moindre mesure, du fait de l'expansion du plasma à la surface des miroirs plasmas à l'échelle de quelques picosecondes. D’après C. Thaury et al., Nature Physics 3 (2007) 424 - 429.

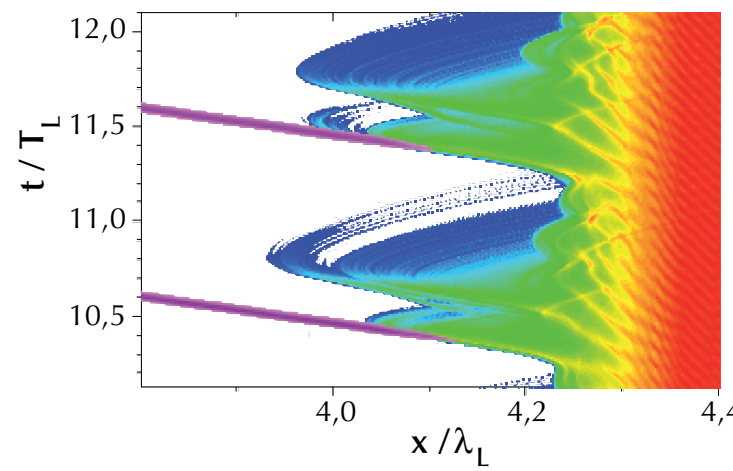

3. Résultat d'une simulation numérique du processus de miroir oscillant relativiste. La carte de couleur allant du rouge au bleu indique la densité électronique du plasma en échelle logarithmique (rouge $=280 \mathrm{n}_{c \text {, }}$ bleu $=0,4 n_{c}$, blanc $\left.<0,4 n_{c}\right)$. Ce plasma est soumis à une impulsion laser d'intensité $1,510^{19} \mathrm{~W} / \mathrm{cm}^{2}$, arrivant en incidence oblique. Sous l'effet du 4,4 champ laser, la surface du plasma effectue un mouvement d'oscillation (anharmonique, du fait des effets relativistes) avec une vitesse pic de l'ordre de c. Ce mouvement relativiste induit un effet Doppler sur le champ réfléchi, qui se traduit par l'émission d'impulsions attosecondes de haute fréquence, aux moments précis où le plasma va vers le vide. Les droites en magenta correspondent aux impulsions attosecondes émises, et sont obtenues en appliquant un filtrage en fréquence au champ électromagnétique total calculé par le code. La fenêtre en temps représentée ici correspond à un zoom sur deux cycles optiques du laser. $T_{L}$ et $\lambda_{L}$ sont respectivement la période optique (typiquement 2,6 fs) et la longueur d'onde (typiquement $800 \mathrm{~nm}$ ) du laser.

\section{Le régime d'interaction relativiste} Encadré 3

Considérons un électron libre dans une onde électromagnétique plane polarisée linéairement, de pulsation $\omega_{\mathrm{L}}$, dont les amplitudes d'oscillation des champs électrique $\mathbf{E}(\mathrm{t})$ et magnétique $B(t)$ sont respectivement $E_{0}$ et $B_{0}=E_{0} / c$. La force s'exerçant sur l'électron est $\mathbf{F}=-\mathrm{e}(\mathbf{E}+\mathbf{v} \times \mathbf{B})$. Lorsque le mouvement de l'électron met en jeu des vitesses $v<<c$, on peut négliger la composante magnétique de cette force. L'électron oscille alors simplement de façon sinusoïdale le long de la direction du champ $\mathbf{E}$, avec une vitesse maximale donnée par

$$
\mathrm{v}_{\max }=\mathrm{eE}_{0} / \mathrm{m} \omega_{\mathrm{L}} \text {. }
$$

Pour un champ laser dans le proche infrarouge $\left(\lambda=800 \mathrm{~nm}, \omega_{\mathrm{L}}=2,310^{15} \mathrm{rad} / \mathrm{s}\right)$ et pour une amplitude de champ de l'ordre de $\mathrm{E}_{0}=4 \mathrm{TV} / \mathrm{m}$ (correspondant à une intensité $\left.\mathrm{I}=210^{18} \mathrm{~W} / \mathrm{cm}^{2}\right), \mathrm{v}_{\max }$ devient de l'ordre de $c$. Ce calcul élémentaire de la vitesse n'est alors plus valide, d'une part parce que la composante $\mathbf{v} \mathbf{x} \mathbf{B}$ ne peut plus être négligée, et d'autre part parce que la variation de masse de l'électron doit être prise en compte. On parle alors de régime d'interaction relativiste. Du fait de ces deux effets, un électron libre dans une onde laser d'une telle intensité effectue alors un mouvement fortement anharmonique le long de la direction de polarisation, et est simultanément poussé dans la direction de propagation du laser sous l'effet du champ B.

\section{>>}

Du fait de la technologie laser utilisée, les impulsions ultra-brèves sont toujours précédées et suivies d'un fond de lumière parasite. Appelé piédestal temporel, celui-ci est beaucoup plus faible que l'impulsion principale - d'un facteur typiquement $10^{6}$ à $10^{9}$, nommé contraste temporel - mais également beaucoup plus long - généralement dans la gamme des nanosecondes (fig. 2). Lorsqu'une telle impulsion est focalisée à ultra-haute intensité sur une cible solide, ce piédestal peut devenir largement assez intense pour ioniser la cible bien avant l'impulsion principale. À titre d'exemple, lorsqu'une impulsion ultra-brève est focalisée à une intensité de $10^{19} \mathrm{~W} / \mathrm{cm}^{2}$, un contraste temporel de $10^{6}$ correspond à un piédestal d'intensité $10^{13} \mathrm{~W} / \mathrm{cm}^{2}$ pendant plusieurs centaines de picosecondes. Cela est bien supérieur au seuil d'ionisation de n'importe quelle cible solide.

Le plasma créé par un tel piédestal a le temps de se détendre avant l'arrivée de l'impulsion principale, qui interagit alors, non plus avec une cible solide pour former un miroir plasma, mais avec un plasma de densité beaucoup plus faible $\left(n \leq n_{c}\right)$ et très inhomogène spatialement. Le contraste insuffisant des lasers ultra-brefs a ainsi longtemps limité les intensités maximales pouvant être utilisées sur miroir plasma.

\section{Les miroirs plasmas comme commutateurs optiques ultrarapides}

Différentes approches ont été étudiées par de nombreux laboratoires pour améliorer le contraste temporel de ces lasers de plusieurs ordres de grandeur. De façon un peu inattendue, l'une des solutions les plus efficaces à ce jour, consiste à utiliser des miroirs plasmas, non plus comme objet d'étude mais comme outil, dans une configuration bien particulière où ils agissent comme des commutateurs optiques ultrarapides à grande dynamique [3].

L'idée est de focaliser le faisceau laser à une intensité modérée sur une cible solide transparente, dont l'interface avec le vide présente initialement une très faible réflectivité $\mathrm{R}_{\mathrm{i}}$ - par exemple, une surface munie d'un traitement diélectrique multicouche anti-reflet à la longueur d'onde du laser. On choisit ces conditions de focalisation de telle sorte que le piédestal soit trop faible pour affecter cette cible : ce piédestal est donc essentiellement transmis à travers la cible 
(à hauteur de plus de 99,5 \% pour un traitement antireflet).

En revanche, la focalisation doit être suffisamment forte pour que l'impulsion principale crée, via des effets non linéaires tels que l'absorption multiphotonique, un plasma dense en surface - ce qui requiert des intensités modérées de l'ordre de $10^{14}-10^{15} \mathrm{~W} / \mathrm{cm}^{2}$. Cette ionisation de la cible se fait de façon extrêmement rapide, en quelques cycles optiques seulement, et le plasma résultant a un coefficient de réflectivité $\mathrm{R}_{\mathrm{f}}$ de l'ordre de $70 \%$.

Dans ces conditions d'interaction, le miroir plasma va donc agir comme un commutateur optique ultrarapide, et réduire le piédestal précédant l'impulsion principale d'un facteur $R_{f} / R_{i}$, soit typiquement plus de deux ordres de grandeur. L'utilisation de deux miroirs plasmas en série (fig. 1, p. 14) permet ainsi un gain de 4 ordres de grandeur sur le contraste (fig. 2), au prix d'une perte de $50 \%$ de l'énergie de l'impulsion principale. Comme déjà souligné, le caractère ultrabref de l'impulsion est essentiel pour éviter une dégradation des propriétés spatiales du faisceau laser sous l'effet de l'expansion du plasma, qui met en jeu des temps de l'ordre de plusieurs picosecondes.

\section{Génération d'harmoniques et d'impulsions attosecondes sur miroir plasma}

Une fois qu'une impulsion de très haut contraste a été obtenue par cette méthode, elle peut être focalisée sur un nouveau miroir plasma, cette fois-ci beaucoup plus fortement et donc à beaucoup plus haute intensité, jusqu'au régime d'interaction relativiste. Dans ce régime, le champ laser induit un mouvement d'oscillation de la surface du miroir plasma, à la fréquence du laser, et ce mouvement met en jeu des vitesses pic de l'ordre de $c$. Ce mouvement relativiste périodique de la surface du miroir induit, par effet Doppler, une distorsion temporelle périodique de l'onde réfléchie par le miroir. $\mathrm{Du}$ fait de cette distorsion périodique, le spectre de la lumière réfléchie est constitué, en plus de la fréquence laser incidente, d'un grand nombre d'harmoniques d'ordre élevé de cette fréquence (fig. 4a). Ce processus dit du "miroir oscillant relativiste ", simple en principe, s'observe clairement dans les simulations numériques (fig. 3). Un tel effet Doppler est l'un des ingrédients essentiels de cette nouvelle optique relativiste [4].
L'une des motivations des recherches sur les miroirs plasmas est la perspective d'atteindre des ordres harmoniques suffisamment élevés pour obtenir des impulsions ultrabrèves cohérentes de rayons $\mathrm{X}$ avec, à la clé, de multiples applications scientifiques. Expérimentalement, la gamme de l'extrême UV (XUV) - typiquement $\lambda \geq 20 \mathrm{~nm}-$ peut d'ores et déjà être atteinte avec des lasers ultra-brefs délivrant quelques joules par impulsion (fig. 4a). Les premières observations expérimentales d'harmoniques dans le domaine des rayons $\mathrm{X}$ ont été effectuées sur des installations lasers délivrant plusieurs centaines de joules, avec des durées d'impulsions plus longues (quelques centaines de fs). La qualité des faisceaux de lumière obtenus est très encourageante pour la suite : des faisceaux bien collimatés sont observés (fig. 4b), et plusieurs sources mutuellement cohérentes ont pu être générées (fig. 4c). Il est donc aujourd'hui prouvé que ces impulsions XUV héritent des propriétés intrinsèques de cohérence spatiale du laser excitateur.

\section{Vers une source attoseconde de $2^{\mathrm{e}}$ génération grâce aux miroirs plasmas}

Un autre intérêt majeur des impulsions ultra-brèves est qu'elles peuvent être utilisées pour étudier la dynamique de systèmes en évolution très rapide. Ainsi, les impulsions femtosecondes sont suffisamment courtes pour résoudre temporellement la dynamique du mouvement des noyaux dans des molécules ou des solides excités. Aujourd'hui, les chercheurs tentent d'étendre ces méthodes à l'étude de la dynamique des électrons dans la matière. Cette dynamique est beaucoup plus rapide que celle des noyaux : l'échelle de temps mise en jeu est typiquement la période de Bohr classique d'un électron dans l'atome d'hydrogène, soit 152 as. Des impulsions plus courtes, dans la gamme attoseconde (1 as $\left.=10^{-18} \mathrm{~s}\right)$, sont donc nécessaires pour étudier ces phénomènes.

Cette gamme de durées a enfin pu être atteinte au début des années 2000 [5] : des impulsions de quelques dizaines d'attosecondes ont été obtenues en utilisant la génération d'harmoniques par interaction laser-atome à intensité modérée $\left(10^{13}-10^{15} \mathrm{~W} / \mathrm{cm}^{2}\right)$ dans un gaz; elles constituent aujourd'hui les impulsions de lumière cohérente les plus brèves jamais produites. Depuis quelques années, on
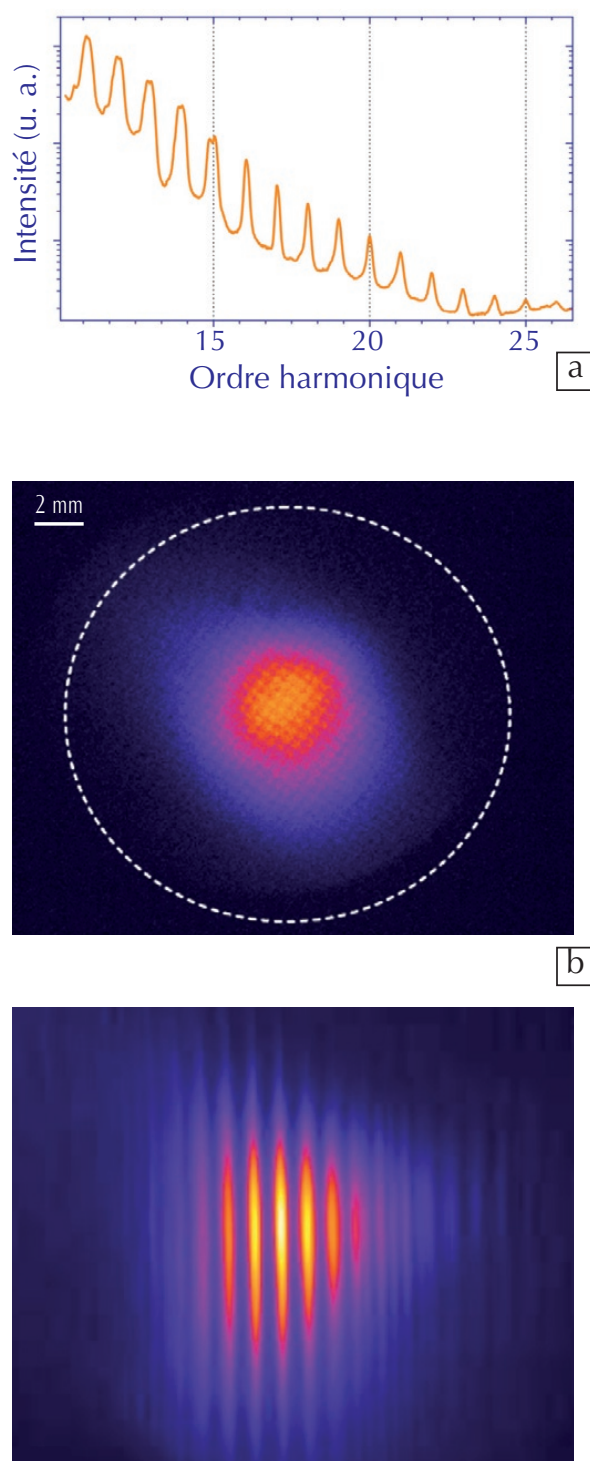

4. Résultats expérimentaux sur la génération d'harmoniques sur miroir plasma. Le graphe (a) présente un spectre obtenu avec le laser UHI10 du CEA Saclay, à une intensité de $10^{19} \mathrm{~W} / \mathrm{cm}^{2}$ sur une cible de plastique. Le nombre d'harmoniques générés est de l'ordre de $4 \gamma^{2}$, où $\gamma$ est le facteur de Lorentz du miroir plasma en mouvement relativiste. Les harmoniques d'ordre $n>15$ sont induites par le mécanisme de miroir oscillant relativiste décrit dans le texte. Pour $n \leq 15$, un autre mécanisme, appelé Coherent Wake Emission (CWE), est dominant. L'image (b) présente le profil transverse d'intensité d'un faisceau d'harmoniques (WE, qui est moins divergent que le faisceau laser initial (limites montrées en pointillés). L'image (c) montre à nouveau ce faisceau, dans le cas où trois taches focales lasers mutuellement cohérentes sont envoyées sur le miroir plasma : le processus de génération d'harmoniques (WE conserve les propriétés de cohérence initiales de la source laser. (D’après C. Thaury et al., Nature Physics 3 (2007) 424-429 et Nature Physics 4 (2008) 631.) 

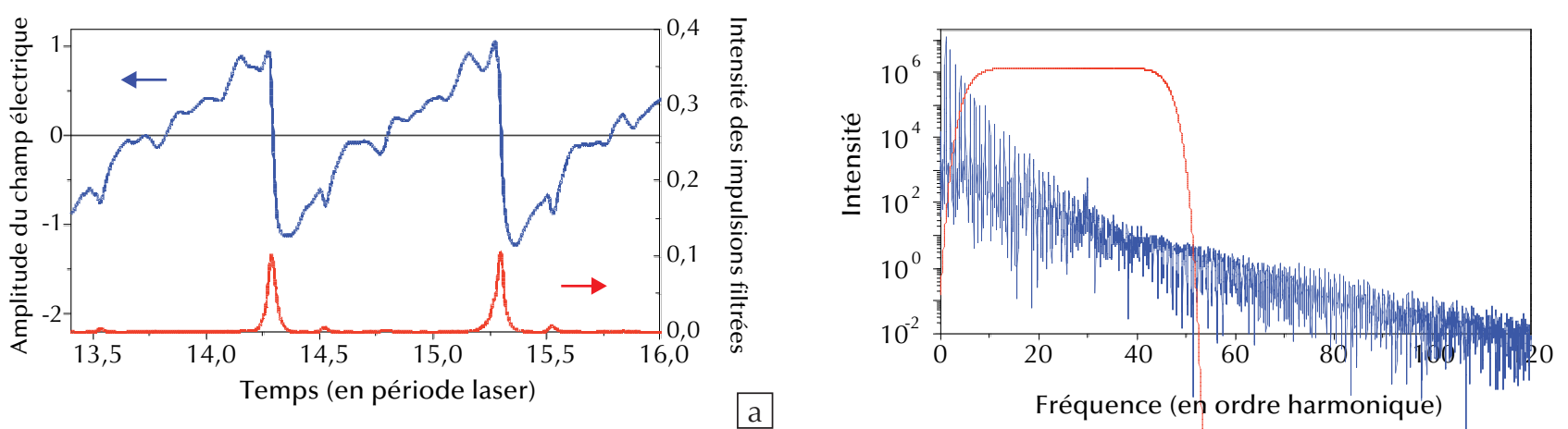

5. Simulation numérique de la génération d’impulsions attosecondes. (a) Amplitude du champ électrique réfléchi (courbe bleue) par un miroir plasma dans le cas de la génération d'harmoniques par effet Doppler (zoom sur deux cycles optiques). Le champ incident est sinusoïdal. L'effet Doppler induit sur le champ réfléchi se traduit par une forme en dent de scie de ce champ. Les conditions physiques sont les mêmes que dans la figure 3. La courbe rouge correspond au profil d'intensité du train d'impulsions attosecondes, obtenu en filtrant un groupe d'harmoniques dans le spectre. Ce spectre est présenté en (b), ainsi que le filtre (courbe rouge) utilisé pour obtenir ce train.

\section{\〉}

commence à les utiliser pour sonder la matière. Cependant, pour étendre la gamme des phénomènes accessibles, il est aujourd'hui essentiel d'obtenir des sources plus intenses, plus brèves et à plus courtes longueurs d'onde.

En effet, les milieux gazeux présentent une restriction majeure : ils cessent d'émettre dès que le gaz est trop fortement ionisé, c'est-à-dire lorsque l'intensité laser dépasse quelques $10^{14} \mathrm{~W} / \mathrm{cm}^{2}$. On ne peut donc pas tirer parti des intensités lasers supérieures à $10^{20} \mathrm{~W} / \mathrm{cm}^{2}$ aujourd'hui disponibles. Au contraire, les miroirs plasmas, constitués d'un milieu déjà fortement ionisé, peuvent fonctionner à des intensités arbitrairement élevées : c'est pourquoi leur utilisation constitue une voie prometteuse pour contourner ce problème et engendrer d'excellentes sources attosecondes « de deuxième génération " [6]. Dans ce cas, les lasers utilisés n'ont plus à être "bridés ", et on peut ainsi obtenir des sources beaucoup plus énergétiques : même en supposant des rendements de conversion à peu près similaires, un gain en énergie de six ordres de grandeur doit en résulter.

Pour produire ces impulsions attosecondes, il suffit de filtrer spectralement la lumière réfléchie par le miroir : le champ électromagnétique obtenu, en éliminant la fréquence fondamentale et en sélectionnant un grand nombre d'harmoniques, correspond uniquement à la distorsion induite par le miroir sur le champ laser. Si cette distorsion est bien localisée temporellement au sein de chaque cycle optique du laser - et il se trouve que cela est bien le cas avec les miroirs plasmas - on obtient alors un train d'impulsions attosecondes extrêmement brèves (fig. 5).

\section{Conclusion : vers l'optique à haute intensité dans les plasmas}

L'optique à ultra-haute intensité est un sujet en plein essor, dynamisé par les progrès constants des lasers. Il n'existe pas, à l'heure actuelle, de théorie générale simple décrivant ce régime de l'optique.

Ainsi, dans le cas des miroirs plasmas, la loi donnant l'ordre harmonique maximum en fonction de l'intensité laser n'est pas encore déterminée. Au-delà de son intérêt fondamental, ce régime est très prometteur pour obtenir de nouvelles sources de lumière : en plus des miroirs plasmas, d'autres « optiques plasmas " fonctionnant à très haute intensité pourraient notamment être utilisées pour amplifier des lasers ultraintenses, mettre en forme ou comprimer temporellement des impulsions ultra-brèves jusqu'à des durées de quelques cycles optiques seulement, ou générer du rayonnement cohérent dans une gamme allant du terahertz aux rayons X.

À titre d'exemple, les simulations montrent qu'une impulsion laser ultra-intense, focalisée sur une feuille de matière suffisamment mince (typiquement $10 \mathrm{~nm}$ ), peut induire un mouvement uniforme relativiste de l'ensemble de cette feuille. On obtient ainsi un miroir relativiste, sur lequel peut se réfléchir une seconde impulsion laser, qui subit alors un effet Doppler uniforme d'un facteur $4 \gamma^{2}$ (où $\gamma$ est le facteur de Lorentz de la feuille)[7]. Si l'on parvient à atteindre des valeurs suffisamment élevées de $\gamma$, cette deuxième impulsion sera alors à la fois comprimée temporellement jusqu'à la gamme attoseconde, et convertie en fréquence dans la gamme des rayons $\mathrm{X}$.
À travers le travail résumé dans cet article, nous espérons avoir montré que l'utilisation des plasmas, à la fois comme objets d'étude fondamentale et comme éléments optiques laser, est une voie très prometteuse pour explorer de nouvelles frontières de l'interaction laser-matière à haute intensité et de la physique attoseconde.

Remerciements

Ce projet a reçu le soutien financier de l'ANR (projet BLAN063134072), du Conseil régional de l'Île-de-France (financement SESAME) et du Conseil général de l'Essonne (financement ASTRE). Les calculs numériques sont réalisés grâce à une allocation de temps de calcul du GENCI, sur les centres de calcul CCRT, IDRIS et CINES.

\section{Références}

$1 \cdot$ P. Balcou et D. Hulin, « Des attosecondes au petawatt, la lumière extrême ", Bulletin de la SFP hors série / Le BUP 875 (2005) 11.

2 - G. Mourou et al., "Optics in the relativistic regime", Rev. Mod. Phys. 78 (2006) 309.

3 - G. Doumy et al., "Complete characterization of a plasma mirror for the production of high-contrast ultra-intense laser pulses", Physical Review E 69 (2004) 26402.

4 - C. Thaury et al., "Plasma mirrors for ultrahighintensity Optics", Nature Physics 3 (2007) 424.

- F. Krausz et M. Ivanov, "Attosecond physics", Rev. Mod. Phys. 81 (2009) 163.

6• F. Quéré, “Ultrafast science: Attosecond plasma optics", Nature Physics (News and Views paper) 5 (2009) 93.

7 - T. Esirkepov et al., "Boosted High-Harmonics Pulse from a Double-Sided Relativistic Mirror", Phys. Rev. Lett. 103 (2009) 025002. 